\title{
Topical Photodynamic Therapy Using Different Porphyrin Precursors Leads to Differences in Vascular Photosensitization and Vascular Damage in Normal Mouse Skin
}

\author{
Tom A. Middelburg ${ }^{1}$, Hannah C. de Vijlder ${ }^{2}$, Henriette S. de Bruijn ${ }^{3}$, Angélique van der Ploeg-van den \\ Heuvel $^{3}$, H. A. Martino Neumann ${ }^{1}$, Ellen R. M. de Haas ${ }^{1}$ and Dominic J. Robinson* ${ }^{\star 1,3}$ \\ ${ }^{1}$ Department of Dermatology, Erasmus MC, Rotterdam, The Netherlands \\ ${ }^{2}$ Department of Dermatology, Isala Klinieken, Zwolle, The Netherlands \\ ${ }^{3}$ Department of Radiation Oncology, Center for Optical Diagnostics and Therapy, Department of Otolaryngology and Head \\ and Neck Surgery, Erasmus MC, Rotterdam, The Netherlands
}

Received 20 November 2013, accepted 11 March 2014, DOI: 10.1111/php.12271

\begin{abstract}
Different distributions of hexyl aminolevulinate (HAL), aminolevulinic acid (ALA) and methyl aminolevulinate (MAL) in the superficial vasculature are not well studied but they are hypothesized to play an important role in topical photodynamic therapy (PDT). The colocalization of fluorescent CD31 and protoporphyrin IX (PpIX) was calculated using confocal microscopy of mouse skin sections to investigate the vascular distribution after topical application. Vascular damage leads to disruption of the normal endothelial adherens junction complex, of which CD144 is an integral component. Therefore, normal CD31 combined with loss of normal fluorescent CD144 staining was visually scored to assess vascular damage. Both the vascular PpIX concentration and the vascular damage were highest for HAL, then ALA and then MAL. Vascular damage in MAL was not different from normal contralateral control skin. This pattern is consistent with literature data on vasoconstriction after PDT, and with the hypothesis that the vasculature plays a role in light fractionation that increases efficacy for HAL and ALA-PDT but not for MAL. These findings indicate that endothelial cells of superficial blood vessels synthesize biologically relevant PpIX concentrations, leading to vascular damage. Such vascular effects are expected to influence the oxygenation of tissue after PDT which can be important for treatment efficacy.
\end{abstract}

Abbreviations: ALA, aminolevulinic acid; HAL, hexyl aminolevulinate; MAL, methyl aminolevulinate; PDT, photodynamic therapy; PpIX, protoporphyrin IX.

\section{INTRODUCTION}

In skin photodynamic therapy (PDT) both aminolevulinic acid (ALA) and its methyl ester methyl aminolevulinate (MAL) are being used worldwide for treating malignant and premalignant skin disease. Another ester derivative of ALA is hexyl aminolevulinate (HAL). HAL ointment is registered for photodiagnosis of, and for margin establishment during surgery of bladder cancer

*Corresponding author email: d.robinson@erasmusmc.nl (Dominic J. Robinson) (C) 2014 The American Society of Photobiology but research is ongoing investigating its therapeutic use in the skin, cervix and bladder (1-6). HAL, ALA and MAL are porphyrin precursors that lead to intramitochondrial accumulation of protopophyrin IX, but they have different pharmacokinetic properties. These properties are in part related to the more lipophilic character of HAL and MAL compared to ALA, but are also related to known differences in cellular uptake mechanisms (7-9). This will lead to differences in penetration and biodistribution between them after topical application, which may account for otherwise unexplained differences in biological behavior. In particular the biodistribution in the superficial vasculature could be important to account for these differences. It is known that prolonged topical ALA application results in systemic distribution of PpIX, whereas PpIX is restricted to the site of MAL application in mice $(10,11)$. Furthermore, subcutaneous arterioles completely constricted directly after topical HAL and ALA-PDT of mouse skin (12), whereas this was less frequent for MAL-PDT (13). Related to this, in vitro data show that HAL is more potent than ALA, which in turn is more potent than MAL in photosensitizing human endothelial cells (14). Also, there is more dermal edema after light-fractionated PDT in ALA than MAL: an indirect indication that leakage through the dermal vessel walls is an acute response to light-fractionated ALA-PDT (15). These and other studies (15-17) have led our group to hypothesize that the vasculature also plays a critical role in the mechanism behind the response to light fractionation. Light fractionation involves delivery of a small first light fraction, followed by a dark interval of $2 \mathrm{hr}$ and a larger second light fraction. It improves the preclinical and clinical response of ALA-PDT, but not of MAL-PDT in mouse skin $(16,18)$. Recent data suggest that light fractionation is also beneficial for HAL-PDT (19). We postulate that these differences in response to light fractionation are related to differences in vascular sensitization between the three porphyrin precursors.

However, there remain areas of ambiguity. First, it has not been studied if and to what extent the blood vessels in the superficial dermis accumulate PpIX. Second, it remains unknown if PDT using porphyrin precursors damages these vessels, as opposed to causing transitory vasoconstriction which subsequently recovers. For these reasons we conducted this study, performed on normal mouse skin. The study has two parts: a study of the vascular distribution of PpIX in dermal blood vessels after 
topical HAL, ALA or MAL application (part A), and a study of the vascular damage for each porphyrin precursor after PDT (part B). For both part A and B, the vasculature was visualized in frozen sections of skin biopsies using fluorescent CD31 (platelet endothelial cell adhesion molecule) antibodies, a commonly used marker for this purpose. Confocal microscopy images were subsequently analyzed to calculate the colocalization of PpIX and CD31 as a measure for the distribution of PpIX in endothelial cells (part A). To study the presence and the extent of vascular damage to these superficial blood vessels after light-fractionated PDT (part B), a combination of fluorescent-labeled CD31 and CD144 antibodies were used. The integrity of the vasculature was imaged and scored using a confocal microscope. CD144vascular endothelial cadherin-is an integral component of the endothelial adherens junction complex. Endothelial damage results in a disruption of the normal endothelial complex so that CD144 can no longer be stained along the plasma membrane. This effect is maximal after $24 \mathrm{hr}$, after which CD144 is slowly restored $(20,21)$.

\section{MATERIALS AND METHODS}

A: The vascular distribution of PpIX, $4 \mathrm{hr}$ after the application of HAL, ALA or MAL (the time-point at which light fractionated PDT is performed as described in B). Animals and drug delivery. A total of 20 SKH1-hr mice (Charles River, Someren, The Netherlands) were divided in four groups of five mice. In the first three groups different porphyrin precursors were applied on a $7 \mathrm{~mm}$ skin area: $5 \%$ HAL ointment, $16 \%$ MAL cream or 20\% ALA gel. The fourth group was the control group in which only the gel was applied. The HAL ointment and MAL cream were the standard commercially available products from Photocure (Oslo, Norway) and Galderma (Freiburg Germany), respectively. The ALA gels were freshly prepared by dissolving ALA (Fagron, Oud Beijerland, The Netherlands) in 3\% carboxymethylcellulose in water with $\mathrm{NaOH}$ added to reach $\mathrm{pH} 4$.

Tissue sampling and imaging. After $4 \mathrm{hr}$ occlusion a $5 \mathrm{~mm}$ punch biopsy was taken from the center of the application area and snap frozen in liquid nitrogen. On the day before fluorescence imaging 3 frozen sections $(10 \mu \mathrm{m})$ from each biopsy were cut under subdued light conditions to prevent PpIX photobleaching. In order not to wash out the PpIX fluorescence we chose to minimize the staining procedure and to omit the fixation step. Sections were carefully washed with a few drops of PBS and incubated with rat anti-mouse CD31 conjugated with Alexa Fluor ${ }^{\circledR}$ 488 (BioLegend, Uithoorn, The Netherlands) diluted 1:200 in PBS overnight at $4^{\circ} \mathrm{C}$. Sections were rewashed carefully with PBS and covered with glycerol (1:3 in PBS).

The distributions of PpIX and AlexaFluor (CD31) in the dermis were imaged at a resolution of $512 \times 512$ pixels using a confocal microscope (Zeiss, LSM 510 Meta) in lambda mode at $400 \times$ magnification. PpIX was imaged first utilizing $405 \mathrm{~nm}$ excitation light and spectral detection between 582 and $753 \mathrm{~nm}$. Immediately thereafter Alexa Fluor ${ }^{\circledR} 488$ was imaged under $488 \mathrm{~nm}$ excitation and a band pass filter (BP 530/600) or spectral detection between 493 and $753 \mathrm{~nm}$. From each section three 3 areas were imaged in which blood vessels were clearly visible, using a slice thickness of $5 \mu \mathrm{m}$. The images were exported and analyzed using ImageJ software (version 1.46, National Institute of Health, USA).

An important consideration in this type of approach is artifacts, normally termed bleed-through/overlap, caused by a significant background contribution of each fluorophore in the emission band of the other fluorophore. Preliminary experiments using control samples and singularly stained sections showed that there is no influence from PpIX fluorescence in the detected emission band of AlexaFluor ${ }^{\circledR} 488$ (BP 530-600 nm) and a negligible influence of the tail of AlexFluor ${ }^{\circledR} 488$ fluorescence in the detected emission band of PpIX (620-710 nm) under $405 \mathrm{~nm}$ excitation. The contribution of autofluorescence in both emission bands was accounted for by performing a background subtraction based on an average uniform autofluorescence collected from control sections containing only PpIX or AlexFluor ${ }^{\circledR} 488$ from the region of interest within which colocalization analyses were performed.
Colocalization analysis. Our primary outcome was the colocalization between PpIX and AlexaFluor in the vessel wall. To calculate the extent of colocalization, regions of interest were drawn following all visible vessel walls. In these regions of interest the fluorescence intensities of PpIX and Alexa Fluor were measured. The colocalization was calculated in two ways. First, the degree of correlation between theCD31 and PpIX fluorescence intensities for each pixel in the region of interest were calculated and expressed as the intensity Pearson's correlation coefficient. Second, Mander's coefficients were calculated over the same region of interest, which are another measure for the degree of colocalization (22). This analysis yields two outcomes: M1 and M2. M1 is the proportion of PpIX positive pixels that also contain a CD31 signal intensity larger than zero. M2 is the proportion of CD31 positive pixels that also contain a PpIX signal intensity larger than zero.

B: Vascular damage after light-fractionated $H A L, A L A$ or $M A L-$ PDT. Animals, drug delivery and light-fractionated PDT. A total of 12 SKH1 HR mice were divided into three groups of four animals. Either HAL ointment, MAL cream or ALA gel was applied to a $7 \mathrm{~mm}$ skin area and occluded for $4 \mathrm{hr}$. Then the area was illuminated using $532 \mathrm{~nm}$ laser light at a fluence rate of $89 \mathrm{~mW} \mathrm{~cm}{ }^{-2}$ to a dose at which $50 \%$ of PpIX was bleached (on average this was approximately $3 \mathrm{~J} \mathrm{~cm}^{-2}$ ). The $89 \mathrm{~mW} \mathrm{~cm}$ was calculated to be equivalent to delivery of $50 \mathrm{~mW} \mathrm{~cm}^{-2}$ at $514 \mathrm{~nm}$, which is what we used in most previous experiments. This calculation is based on the PpIX absorption and photon energy for these wavelengths. The rationale for the $50 \%$ photobleaching dose is explained in more detail elsewhere $(23,24)$. After a dark interval of $2 \mathrm{hr}$ a second illumination was delivered to reach a total dose of $100 \mathrm{~J} \mathrm{~cm}^{-2}$.

Tissue sampling and imaging. At $24 \mathrm{hr}$ after PDT, a $7 \mathrm{~mm}$ biopsy was taken from the treated area and from contralateral control skin, sectioned, stained, imaged and analyzed. Both the staining method and the imaging analysis were different from the methods described in A. Frozen sections were fixated with acetone $(20 \mathrm{~min})$, cellular permeability was increased using $0.5 \%$ triton in $1 \%$ BSA in PBS (30 min) and nonspecific staining was reduced using 5\% BSA in PBS $(60 \mathrm{~min})$. The vessel wall was visualized by staining with anti CD31 conjugated with AlexaFluor ${ }^{\circledR} 647$ (BioLegend, San Diego, CA, USA) in $1 \%$ BSA in PBS. To determine damage to the vessel wall the sections were also stained with anti-CD144 conjugated with biotin in $1 \%$ BSA in PBS. Both CD31 and $\mathrm{CD} 144$ stainings were performed simultaneously at $4^{\circ} \mathrm{C}$ overnight, followed by biotin-specific staining with streptavidin efluor 570 in $1 \%$ BSA in PBS (both from eBioscience, Vienna, Austria) the next morning.

Scoring of vascular damage. In each section three $400 \times$ images were scored for the presence of vascular damage which was our primary outcome for this part of the study. Vascular damage was defined as the absence of an identifiable blood vessel in the CD144 staining while there was a blood vessel visible in the CD31 staining. This scoring method was applied to both the PDT-treated skin and the contralateral control skin. This resulted in 9 scores of vascular damage (yes/no) per mouse for the PDT skin sections and another 9 for the control skin. The investigator was blinded from the treatment groups.

Drug application, taking the biopsies and performing PDT were all performed under general anesthesia. The experiments were approved by the local animal experiments committee.

\section{RESULTS}

\section{Part A: PpIX colocalization with CD31}

There was clear PpIX fluorescence in the epidermis and in hair follicles for all three groups (HAL, ALA, MAL). In the dermis there was much less PpIX signal. Some of the dermal PpIX was visible as spindle-shaped fluorescence throughout the interstitial upper dermis, corresponding to dermal fibroblasts. In many cases the PpIX fluorescence was visibly located in the wall of a superficial blood vessel, which was also positively stained with CD31 (Fig. 1).

Such colocalization of PpIX with CD31 was variable per mouse in all groups. On average the highest colocalization was observed in the HAL group, less in the ALA and MAL groups and the lowest in the control skin (Fig. 2). Both the average 


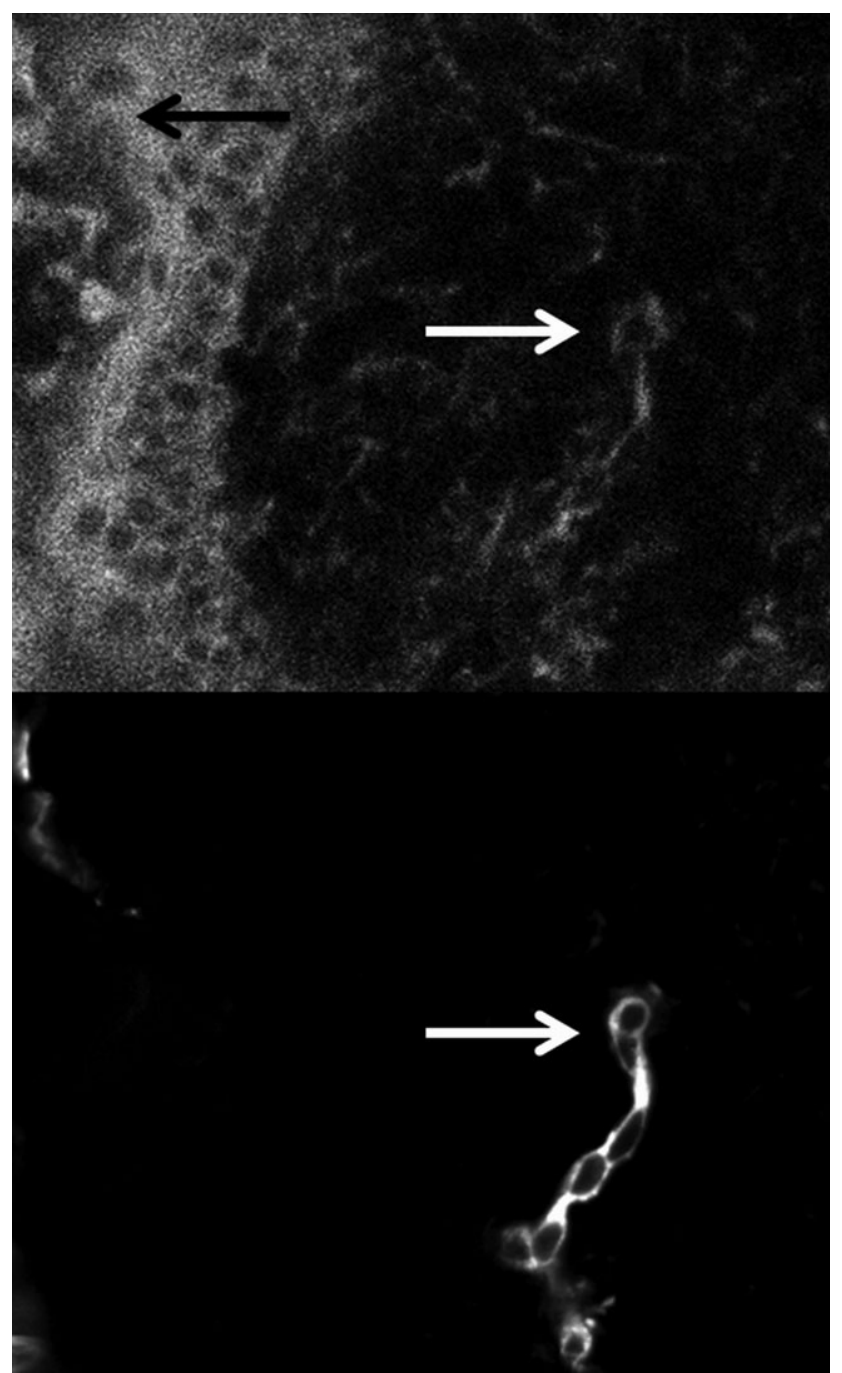

Figure 1. Illustrative example of a PpIX fluorescence image (upper) and the corresponding Alexafluor ${ }^{\circledR} 488$-conjugated CD31 fluorescence image (lower). Note the presence of PpIX in the epidermis (black arrow) and in the endothelium of a dermal blood vessel (white arrows). There was also some nonspecific CD31 staining in the epidermis. Magnification $400 \times$. correlation and M2 coefficients per mouse in the three groups (HAL, ALA and MAL) were significantly different from the control group (correlation coefficient: $P=0.005$; M2: $P=0.002$ ), but not different from each other (correlation coefficient: $P=0.19$; M2: $P=0.29$, Kruskall-Wallis test). The colocalization was significantly higher in the HAL, ALA and MAL when individually compared with the control group $(P=0.008$ for all comparisons except for the M2 coefficient of MAL versus control, $P=0.16$ Mann-Whitney $U$-test). The M1 coefficient was high ( $>0.95$ for all groups).

\section{Part B: vascular damage}

In all three groups (HAL, ALA and MAL) focal loss of normal fluorescent CD144 located along the vascular endothelium was observed. Instead, the CD144 was relocated into the immediate surroundings of the vessel, indicating disruption of the endothelium. Such a response was scored as vascular damage and an example of this is shown in Fig. 3.

This type of loss of identifiable vessel in the CD144 staining was also observed in $400 \times$ images of normal skin (in 12/99 of total images), but much more often in the PDT-treated skin (in $33 / 99$ of total images). The number of total images that was scored as vascular damage was highest in the HAL-PDT group (14/36), then in the ALA-PDT group (10/27) and lowest in the MAL-PDT group (9/36). When the data per mouse (instead of per image) are considered, three of four mice in the HAL group showed more vascular damage in the PDT-treated skin than in normal skin, which was two of three mice in the ALA group and two of four mice in the MAL group.

The percentage of $400 \times$ images that was scored as vascular damage is illustrated in Fig. 4 for all three groups, including PDT treated and normal skin. One mouse in the ALA group died which is why there are fewer data in this group. In both the HAL and ALA-PDT-treated skin the percentage in which vascular damage was scored in $400 \times$ images was statistically significantly higher than the combined data of all 99 control skin images $(P=0.0005$ and $P=0.003$, respectively). In the MAL group there was no significant difference between PDT-treated skin and control skin $(P=0.068$, chi-square test). The number
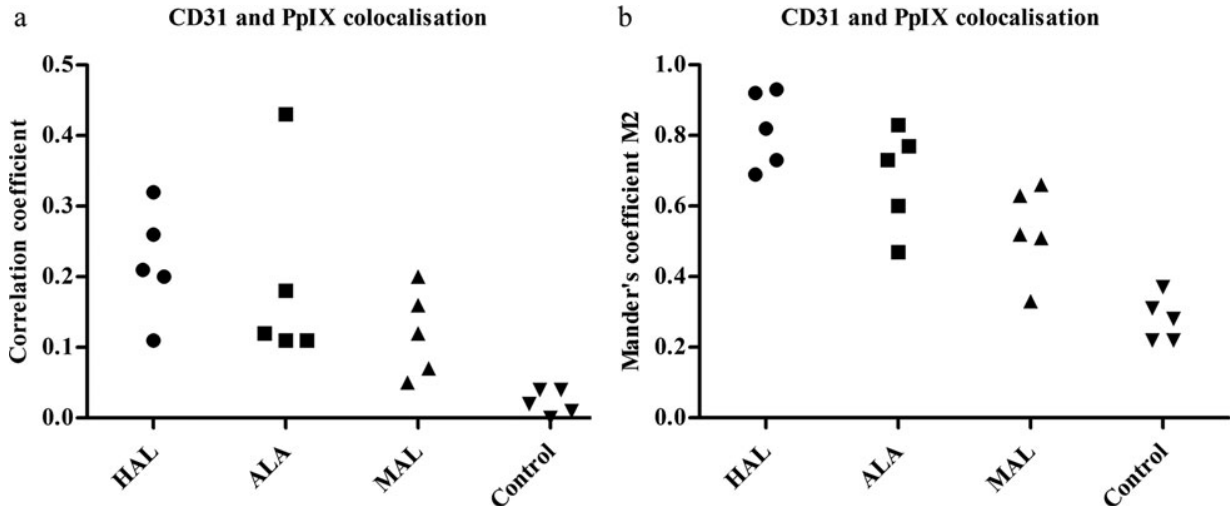

Figure 2. The extent of colocalization between PpIX fluorescence and CD31 conjugated with AlexaFluor ${ }^{\circledR} 488$, represented as (a) the Pearson's correlation coefficient between individual pixels; and (b) Mander's M2 coefficient, which indicates the proportion of CD31 positive pixels that also contain PpIX fluorescence. Data shown are average values per mouse $(n=5)$ for each group. The value for each mouse was calculated from a total of nine images taken in three microscopic tissue sections. 


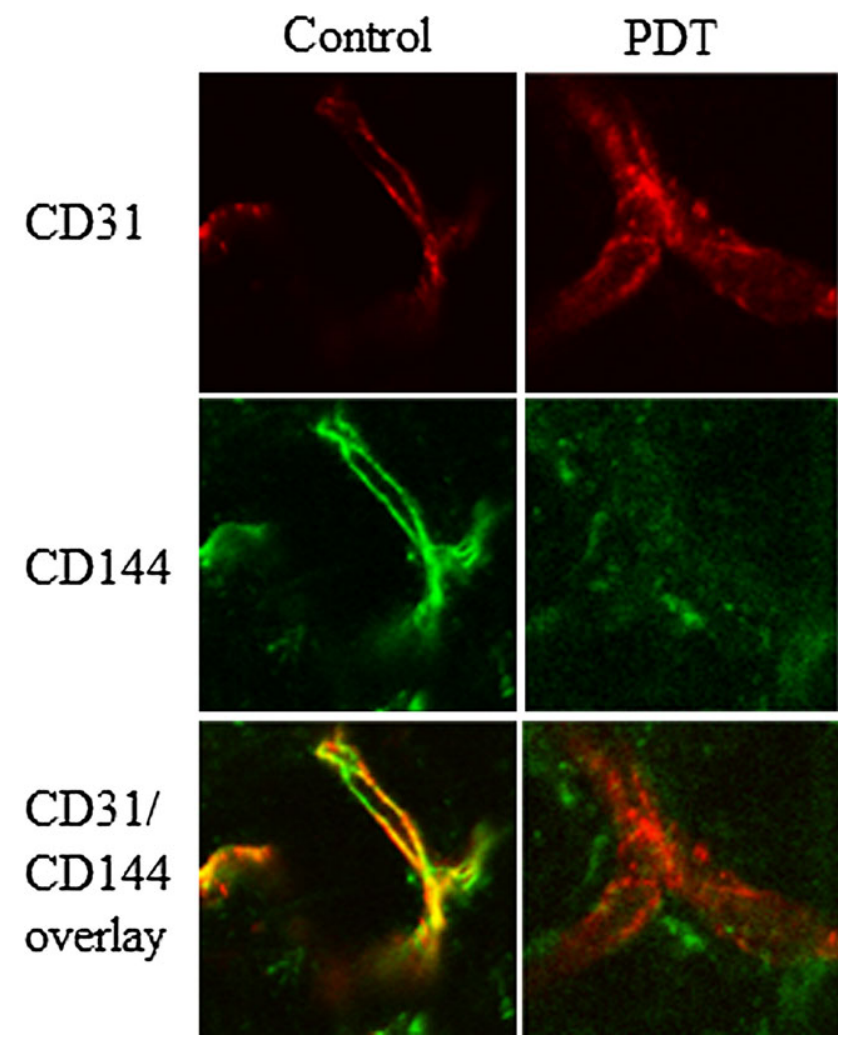

Figure 3. Confocal fluorescence images example of control skin (left) and HAL-PDT-treated skin (right) of the same mouse. The upper images are the CD31 and the corresponding CD144 stainings. The lower image is the overlay of the upper images, showing overlap of CD31/CD144 in the control skin, but not in the PDT skin. In the PDT-treated skin the CD144 no longer stained along the plasma membrane, indicating vascular damage. Magnification $400 \times$, zoom 5.3×.

\section{Extent of vascular damage}

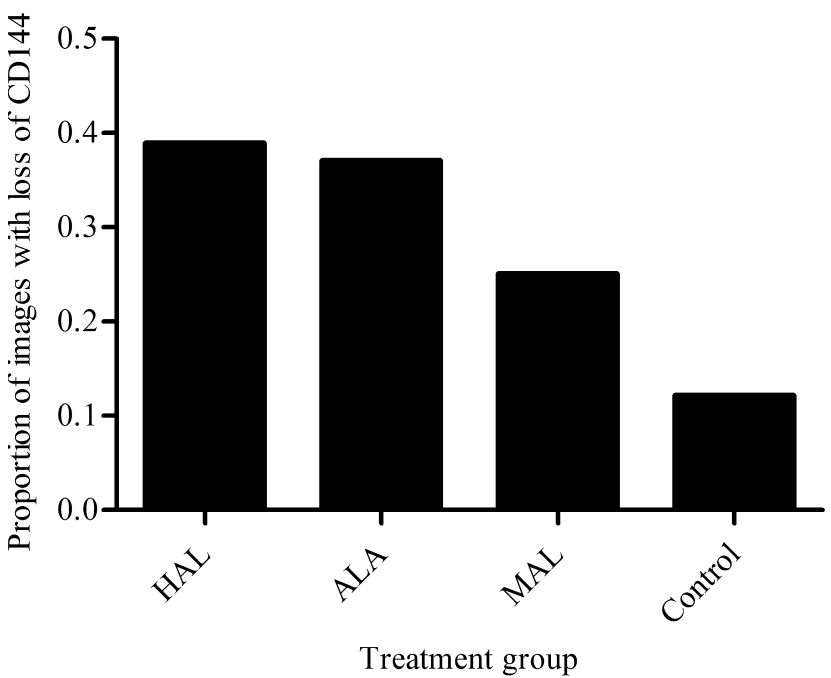

Figure 4. Proportion of $400 \times$ images in which vascular damage was scored for the three PDT-treated groups ( $n=36$ for HAL, $n=27$ for ALA, $n=36$ for MAL and $n=99$ for control). HAL and ALA groups were significantly different from control skin $(P=0.0005$ and 0.003 , respectively, but MAL was not $(P=0.068$, Chi-squared test).

of vascular damage scores per $400 \times$ image was not different between the HAL, ALA and MAL groups $(P=0.408$ chi square test).

\section{DISCUSSION}

In this study, the distribution of PpIX in dermal blood vessels was investigated after either HAL, ALA or MAL application. In addition, we studied if fractionated PDT using HAL, ALA or MAL damages the vascular integrity of these vessels. We found that after application of all three porphyrin precursors, endothelial cells in the vessel walls synthesized PpIX. However, these PpIX concentrations (represented by the degree of colocalization between PpIX and CD31) were highest in the HAL group, next in the ALA group and lowest in the MAL group. The same trend was seen for the vascular damage after PDT: highest vascular damage scores for HAL, followed by ALA and then MAL.

These combined findings indicate that the amount of PpIX synthesis in the vessel wall actually causes vascular damage and that this effect is the strongest for HAL. We previously reported more extensive constriction of subcutaneous arterioles for HAL than for ALA-PDT, which was correlated with PpIX concentration in the vessel wall (12) and more extensive arteriole constriction after PDT using ALA than MAL (13). In another study we observed more acute edema after ALA than MAL-PDT (15). From these studies and our current findings, we can conclude that 5\% HAL ointment leads to higher PpIX concentrations in dermal and subcutaneous vessel walls than 20\% ALA gel, which leads to higher PpIX concentrations than 16\% MAL cream. As a logical result, the vascular constriction and vascular damage is increasingly extensive with this increasing potential to accumulate PpIX in vessel walls.

From our current findings, which were performed on normal skin, it can be assumed that the vasculature is also damaged after topical HAL and ALA-PDT of cancer tissue. Obviously the aim here was to destroy all cancer cells while preserving normal tissue. This ratio may be dependent on the vascular response. When vessels constrict too much during PDT, this limits the oxygen supply to the PDT-treated site. This type of response will decrease the potency of PDT to destroy tumor cells. On the other hand, if vasoconstriction happens late during or after PDT or if vessels are damaged, local ischemia may help destroy cancer cells that would otherwise survive. However, when the ischemia becomes too extensive or long-lasting, normal tissue will die too which may result in an inferior cosmetic or functional outcome. This suggests that there may be a delicate balance between too much or too little vascular photosensitization to achieve optimal PDT effect. The phenomenon that the PDT outcome is related in a complex manner to vascular responses and oxygen supply has already been described for PDT using systemic porphyrins in the 1980s (25-27). Our current results indicate that the extent of vascular constriction or damage may be important for the overall effect of topical PDT too.

In addition to enhanced tumor cell kill due to hypoxia from vascular damage, the vascular responses may also be part of the mechanism behind the success of light-fractionated PDT. For ALA-PDT, light fractionation has proven beneficial in both preclinical $(23,28-30)$ and clinical studies $(18,31)$. We hypothesize that the vascular endothelium synthesizes a small but relevant amount of PpIX which makes it vulnerable to a second illumination. Because the effect of light fractionation is stronger for relatively low PpIX concentrations (17) the vascular endothelium is a potential target for fractionated PDT. In both in vitro and in vivo studies it has been shown that vascular endothelial cells can 
be photosensitized using ALA (14,32). It could very well be, however, that a minimum PpIX concentration is necessary to cause vascular damage. Because light fractionation does not increase efficacy of MAL-PDT in normal mouse skin (16), it may be that the endothelial PpIX concentration is too low which is in accordance with our current findings. Our current data are also in line with the expectation and recent evidence that light fractionation may also be beneficial for HAL-PDT (19), which could be relevant for clinical trials.

Before translating our findings to clinical implications, however, we should bear in mind that such a step needs to be taken carefully. Apart from the obvious biological differences between humans and mice, a considerable difference lies in the increased thickness of human skin (and other epithelial surfaces such as those of the bladder and cervix). This is relevant with respect to penetration. Also, tumor tissue such as basal cell carcinoma is known to have a different vasculature than normal skin $(33,34)$ which potentially influences the ability of the endothelial cells to accumulate PpIX.

Despite these differences between human and mouse tissues, our findings may be useful when designing clinical trials. It could very well be that vascular responses also play a role in clinical PDT, for which there is some evidence in the literature. Simulations of PpIX photobleaching kinetics and subsequent measurements have confirmed blood flow changes during ALA-PDT of basal cell carcinoma $(35,36)$ and blood volume and saturation changes were observed in ALA-PDT of actinic keratosis (37). Also, after PDT for skin rejuvenation the number of telangiectasias in the face decreased after ALA-PDT (38), but not after MALPDT (39). This could reflect the increased ability to sensitize endothelial cells of ALA compared with MAL. ALA-PDT is also used off-label to treat port wine stains (40). It is questionable, however, if the vascular accumulation of PpIX in port wine stains will be sufficient to cause direct vascular damage, or that other mechanisms after PDT may play a role. The effect of ALA-PDT on port wine stains can, however, be considered an indication that the vasculature can also be a primary target of PDT, which suggests that other vascular tumors may theoretically also benefit from topical HAL or ALA-PDT.
There are some limitations to this study which must be addressed. Most importantly, we observed a loss of CD144 staining along the vessel wall also in the normal skin sections. As the normal skin was taken from contralateral site is it extremely unlikely that this finding represents actual vascular damage. It may instead be associated with a background level of loss of CD144 staining that is also observed in other cellular studies $(41,42)$. It can then be logically assumed that such a background level is also present in the PDT-treated sections. Although this decreases the specificity of our scoring system, we did find a significantly larger loss of CD144 staining in the PDT-treated skin than in normal skin using unpaired statistical testing. We are therefore confident in our conclusion that our findings reflect the presence of vascular damage.

Another potential concern is the relatively low Pearson's correlation coefficient, which can be explained by its sensitivity to background noise and to variable fluorescence intensities (22). The M2 coefficient, however, provides additional information on the degree of colocalization that is less sensitive to noise and uneven PpIX intensity distributions in this case. Because our region of interest contains only the vessel wall, it is saturated with CD31 positive pixels. The high value of M2 (especially high for HAL) indicates that a large proportion of these CD31 positive pixels also contain PpIX signal, even if this signal is low. The level of noise is represented by the average M2 value in the control sections (0.28). This high noise level originates from the PpIX images. It explains why the M1 coefficient is almost 1 for all groups as nearly all of the PpIX/noise pixels will colocalize with CD31 pixels in our region of interest. A way to reduce the signal to noise ratio in the future may be to increase the integration times during the PpIX image acquisition. However, it should be noted that the rapid photobleaching rate of PpIX will limit the signal to noise ratio that is achievable.

It must also be noted that we did not include a functional test to study the functional consequences of the vascular damage, which would provide additional evidence for vascular damage. We previously observed more edema after light-fractionated ALA-PDT than after MAL-PDT (15), which indicates increased
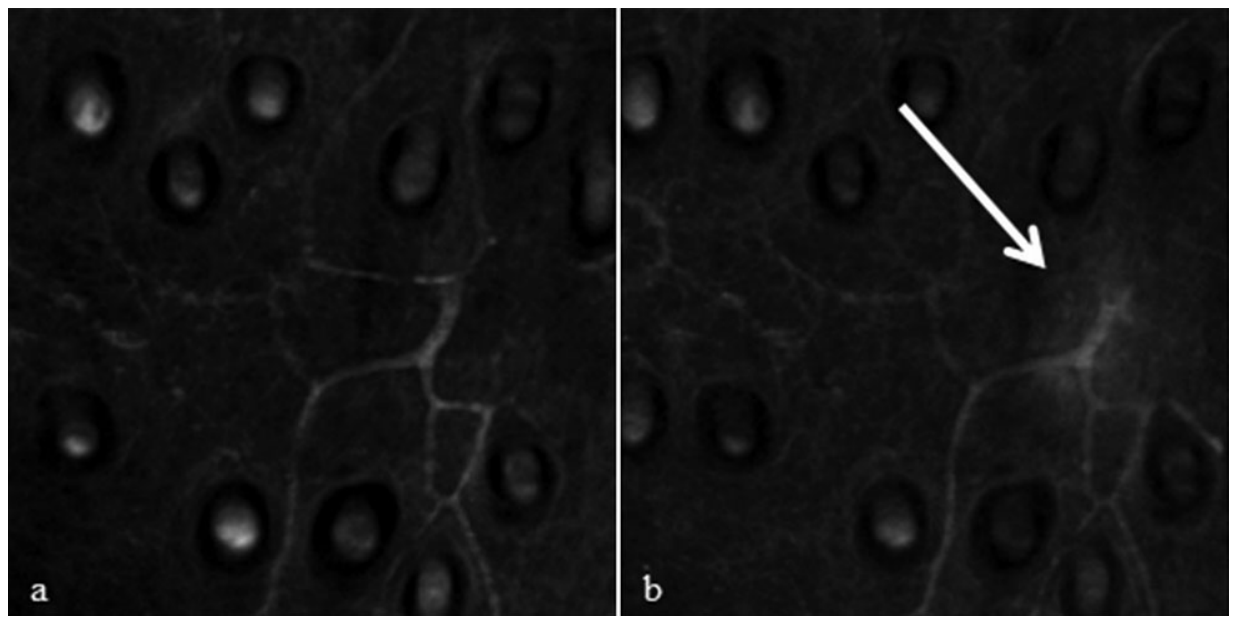

Figure 5. Transepidermal confocal images in a mouse skin fold chamber model. Immediately after fractionated ALA-PDT fluorescein was injected intravenously. On the left (a) is a transmission image before PDT and on the right (b) a fluorescence image after PDT showing leakage of fluorescein into the perivascular space (white arrow). The round dots in both images are hair follicle units. Image adapted from the thesis "New insights into photodynamic therapy using porphyrin precursors" by de Vijlder in 2013 with permission. 
permeability after PDT. To support the results of this study we have performed additional in-vivo intra-vital microscopy investigating the distribution of fluorescein after fractionated ALAPDT. In an extension of the mouse dorsal skin fold chamber model that we have previously used to investigate the vascular response during PDT with porphyrin precursors (12) $514 \mathrm{~nm}$ light from the confocal microscope (ca $12 \mathrm{~J} \mathrm{~cm}^{-2}$ ) was used both for light delivery and for imaging through the epidermis. Immediately after PDT, fluorescein (FITC-BSA, Sigma) was injected intravenously at a dose of $0.2 \mathrm{mg} / \mathrm{mouse}$. Trans-epidermal images were obtained and showed focal leakage of fluorescein into the perivascular space. An example image of this is shown in Fig. 5.

Such focal fluorescein leakage is in accordance with our current finding of focal loss of CD144 staining. Increased permeability leads to stress fiber formation, followed by phosphorylation of RhoA, inhibiting CD144 formation (43). So the direct increase in permeability is a functional measure of vascular dysfunction, which results in structural changes that can be detected as loss of normal CD144 staining $24 \mathrm{hr}$ later.

A possible limitation could also be that we compared the ability of HAL, ALA and MAL to penetrate through the epidermis and synthesize PpIX in the vascular endothelium, but we used different concentrations and formulations. In several studies it has been shown that lower concentrations of HAL than ALA and lower concentrations of ALA than MAL are needed to reach the same PpIX concentration in different models $(6,44-46)$. Also, the vehicle can influence the penetration of the molecules $(47,48)$. To translate the relevance of our findings for clinical applications, we considered it more useful to compare the standard available products which is why we chose to use these in this study.

For future research it may be interesting to investigate if there are any differences in vascular damage after single versus light-fractionated PDT. If our hypotheses are correct we can expect to see less vascular damage after a single illumination, but considering the presence of CD144 staining artifacts, the number of animals and/or sections needs to be larger to detect statistical differences. Another interesting future step would be to further investigate the benefit of light fractionated for HALPDT in vivo.

In conclusion we have now shown for the first time that there is vascular damage of dermal blood vessels after topical lightfractionated HAL and ALA-PDT, which is related to the ability of the vessel wall to synthesize PpIX. In this and previous work, we find a consistent trend that shows the highest vascular PpIX synthesis, vascular constriction and vascular damage for HAL, followed by ALA and then MAL. While the vascular constriction that we found in previous studies may be relevant for the oxygen supply during PDT, the vascular damage that we found in this study could also be relevant for the oxygen supply after light-fractionated PDT. This potentially plays a role as an enhanced kill mechanism of light-fractionated PDT for cancer cells that would otherwise survive. In addition, our findings suggest that topical-fractionated HAL and ALA-PDT can be used to primarily target the vasculature. This could be an important aspect of PDT for cosmetic purposes, port wine stains and possibly other vascular tumors.

Acknowledgement-Funding: The HAL ointment $\left(\mathrm{Cevira}^{\mathrm{TM}}{ }^{\mathrm{M}}\right)$ was supplied by Photocure (Photocure, Oslo, Norway).

\section{REFERENCES}

1. Soergel, P., X. Wang, H. Stepp, H. Hertel and P. Hillemanns (2008) Photodynamic therapy of cervical intraepithelial neoplasia with hexaminolevulinate. Lasers Surg. Med. 40, 611-615.

2. Soergel, P., L. Makowski, E. Makowski, C. Schippert, H. Hertel and P. Hillemanns (2011) Treatment of high grade cervical intraepithelial neoplasia by photodynamic therapy using hexylaminolevulinate may be costeffective compared to conisation procedures due to decreased pregnancy-related morbidity. Lasers Surg. Med. 43, 713720 .

3. Soergel, P., G. F. Dahl, M. Onsrud and P. Hillemanns (2012) Photodynamic therapy of cervical intraepithelial neoplasia 1-3 and human papilloma virus (HMV) infection with methylaminolevulinate and hexaminolevulinate-a double-blind, dose-finding study. Lasers Surg. Med. 44, 468-474.

4. Juzeniene, A., K. P. Nielsen, L. Zhao, G. A. Ryzhikov, M. S. Biryulina, J. J. Stamnes, K. Stamnes and J. Moan (2008) Changes in human skin after topical PDT with hexyl aminolevulinate. Photodiagnosis Photodyn. Ther. 5, 176-181.

5. Bader, M. J., H. Stepp, W. Beyer, T. Pongratz, R. Sroka, M. Kriegmair, D. Zaak, M. Welschof, D. Tilki, C. G. Stief and R. Waidelich (2012) Photodynamic therapy of bladder cancer - a phase I study using hexaminolevulinate (HAL). Urol. Oncol. 20, 20.

6. Dognitz, N., D. Salomon, M. Zellweger, J. P. Ballini, T. Gabrecht, N. Lange, H. van den Bergh and G. Wagnieres (2008) Comparison of ALA- and ALA hexyl-ester-induced PpIX depth distribution in human skin carcinoma. J. Photochem. Photobiol., B 93, 140-148.

7. Rodriguez, L., A. Batlle, G. Di Venosa, S. Battah, P. Dobbin, A. J. Macrobert and A. Casas (2006) Mechanisms of 5-aminolevulinic acid ester uptake in mammalian cells. Br. J. Pharmacol. 147, 825833.

8. Rud, E., O. Gederaas, A. Hogset and K. Berg (2000) 5-aminolevulinic acid, but not 5-aminolevulinic acid esters, is transported into adenocarcinoma cells by system BETA transporters. Photochem. Photobiol. 71, 640-647.

9. Schulten, R., B. Novak, B. Schmitz and H. Lubbert (2012) Comparison of the uptake of 5-aminolevulinic acid and its methyl ester in keratinocytes and skin. Naunyn Schmiedebergs Arch. Pharmacol. 385, 969-979.

10. Juzeniene, A., P. Juzenas, V. Iani and J. Moan (2002) Topical application of 5-aminolevulinic acid and its methylester, hexylester and octylester derivatives: considerations for dosimetry in mouse skin model. Photochem. Photobiol. 76, 329-334.

11. Moan, J., L. W. Ma, A. Juzeniene, V. Iani, P. Juzenas, F. Apricena and Q. Peng (2003) Pharmacology of protoporphyrin IX in nude mice after application of ALA and ALA esters. Int. J. Cancer 103, 132-135.

12. Middelburg, T. A., H. S. de Bruijn, L. Tettero, A. van der Ploeg van den Heuvel Ploeg, H. A. Neumann, E. R. de Haas and D. J. Robinson (2013) Topical hexylaminolevulinate and aminolevulinic acid photodynamic therapy: complete arteriole vasoconstriction occurs frequently and depends on protoporphyrin IX concentration in vessel wall. J. Photochem. Photobiol., B 126, 26-32.

13. de Vijlder, H. C. (2013) New Insights into Photodynamic Therapy using porphyrin precursors. Ph.D. thesis, Erasmus University Rotterdam, Rotterdam, the Netherlands.

14. Rodriguez, L., H. S. de Bruijn, G. Di Venosa, L. Mamone, D. J. Robinson, A. Juarranz, A. Batlle and A. Casas (2009) Porphyrin synthesis from aminolevulinic acid esters in endothelial cells and its role in photodynamic therapy. J. Photochem. Photobiol., B 96, 249254.

15. de Bruijn, H. S., C. Meijers, A. van der Ploeg-van den Heuvel, H. J. Sterenborg and D. J. Robinson (2008) Microscopic localisation of protoporphyrin IX in normal mouse skin after topical application of 5-aminolevulinic acid or methyl 5-aminolevulinate. J. Photochem. Photobiol., B 92, 91-97.

16. de Bruijn, H. S., E. R. de Haas, K. M. Hebeda, A. van der Ploegvan den Heuvel, H. J. Sterenborg, H. A. Neumann and D. J. Robinson (2007) Light fractionation does not enhance the efficacy of methyl 5-aminolevulinate mediated photodynamic therapy in normal mouse skin. Photochem. Photobiol. Sci. 6, 1325-1331. 
17. de Bruijn, H. S., A. G. Casas, G. Di Venosa, L. Gandara, H. J. Sterenborg, A. Batlle and D. J. Robinson (2013) Light fractionated ALA-PDT enhances therapeutic efficacy in vitro; the influence of PpIX concentration and illumination parameters. Photochem. Photobiol. Sci. 12, 241-245.

18. de Haas, E. R., B. Kruijt, H. J. Sterenborg, H. A. Martino Neumann and D. J. Robinson (2006) Fractionated illumination significantly improves the response of superficial basal cell carcinoma to aminolevulinic acid photodynamic therapy. J. Invest. Dermatol. 126, 2679-2686.

19. Middelburg, T., H. De Bruijn, A. Van der Ploeg van den Heuvel, H. Neumann and D. Robinson (2013) The effect of light fractionation with a two-hour dark interval on the efficacy of topical hexyl-aminolevulinate photodynamic therapy in normal mouse skin. Photodiagnosis Photodyn. Ther. 10, 703-709.

20. Sutton, T. A., H. E. Mang, S. B. Campos, R. M. Sandoval, M. C. Yoder and B. A. Molitoris (2003) Injury of the renal microvascular endothelium alters barrier function after ischemia. Am. J. Physiol. Renal Physiol. 285, F191-F198.

21. Corada, M., M. Mariotti, G. Thurston, K. Smith, R. Kunkel, M. Brockhaus, M. G. Lampugnani, I. Martin-Padura, A. Stoppacciaro, L. Ruco, D. M. McDonald, P. A. Ward and E. Dejana (1999) Vascular endothelial-cadherin is an important determinant of microvascular integrity in vivo. Proc. Natl. Acad. Sci. USA 96, 98159820.

22. Bolte, S. and F. P. Cordelieres (2006) A guided tour into subcellular colocalization analysis in light microscopy. J. Microsc. 224, 213232.

23. de Bruijn, H. S., A. van der Ploeg-van den Heuvel, H. J. Sterenborg and D. J. Robinson (2006) Fractionated illumination after topical application of 5-aminolevulinic acid on normal skin of hairless mice: the influence of the dark interval. J. Photochem. Photobiol., B 85, 184-190.

24. Middelburg, T. A., F. Van Zaane, H. S. De Bruijn, A. Van Der Ploeg-van den Heuvel, H. J. Sterenborg, H. A. Neumann, E. R. De Haas and D. J. Robinson (2010) Fractionated illumination at low fluence rate photodynamic therapy in mice. Photochem. Photobiol. 86, $1140-1146$.

25. Henderson, B. W. and V. H. Fingar (1987) Relationship of tumor hypoxia and response to photodynamic treatment in an experimental mouse tumor. Cancer Res. 47, 3110-3114.

26. Henderson, B. W., S. M. Waldow, T. S. Mang, W. R. Potter, P. B. Malone and T. J. Dougherty (1985) Tumor destruction and kinetics of tumor cell death in two experimental mouse tumors following photodynamic therapy. Cancer Res. 45, 572-576.

27. Henderson, B. W. and V. H. Fingar (1989) Oxygen limitation of direct tumor cell kill during photodynamic treatment of a murine tumor model. Photochem. Photobiol. 49, 299-304.

28. Robinson, D. J., H. S. de Bruijn, N. van der Veen, M. R. Stringer, S. B. Brown and W. M. Star (1998) Fluorescence photobleaching of ALA-induced protoporphyrin IX during photodynamic therapy of normal hairless mouse skin: the effect of light dose and irradiance and the resulting biological effect. Photochem. Photobiol. 67, 140149.

29. Robinson, D. J., H. S. de Bruijn, W. J. de Wolf, H. J. Sterenborg and W. M. Star (2000) Topical 5-aminolevulinic acid-photodynamic therapy of hairless mouse skin using two-fold illumination schemes: PpIX fluorescence kinetics, photobleaching and biological effect. Photochem. Photobiol. 72, 794-802.

30. Robinson, D. J., H. S. de Bruijn, W. M. Star and H. J. Sterenborg (2003) Dose and timing of the first light fraction in two-fold illumination schemes for topical ALA-mediated photodynamic therapy of hairless mouse skin. Photochem. Photobiol. 77, 319-323.

31. de Vijlder, H. C., H. J. Sterenborg, H. A. Neumann, D. J. Robinson and E. R. de Haas (2012) Light fractionation significantly improves the response of superficial basal cell carcinoma to aminolaevulinic acid photodynamic therapy: five-year follow-up of a randomized, prospective trial. Acta Derm. Venereol. 92, 641-647.
32. Chang, C. J., S. F. Ma and F. C. Wei (1999) In vitro and in vivo photosensitizing capabilities of 5-ALA in vascular endothelial cells. Changgeng Yi Xue Za Zhi 22, 181-188.

33. Bedlow, A. J., A. W. Stanton, S. Cliff and P. S. Mortimer (1999) Basal cell carcinoma-an in-vivo model of human tumour microcirculation? Exp. Dermatol. 8, 222-226.

34. Grunt, T. W., A. Lametschwandtner and O. Staindl (1985) The vascular pattern of basal cell tumors: light microscopy and scanning electron microscopic study on vascular corrosion casts. Microvasc. Res. 29, 371-386.

35. Wang, K. K., W. J. Cottrell, S. Mitra, A. R. Oseroff and T. H. Foster (2009) Simulations of measured photobleaching kinetics in human basal cell carcinomas suggest blood flow reductions during ALA-PDT. Lasers Surg. Med. 41, 686-696.

36. Becker, T. L., A. D. Paquette, K. R. Keymel, B. W. Henderson and U. Sunar (2010) Monitoring blood flow responses during topical ALA-PDT. Biomed. Opt. Express. 2, 123-130.

37. Middelburg, T. A., S. C. Kanick, E. R. de Haas, H. J. Sterenborg, A. Amelink, M. H. Neumann and D. J. Robinson (2011) Monitoring blood volume and saturation using superficial fibre optic reflectance spectroscopy during PDT of actinic keratosis. J. Biophotonics 4, 721-730.

38. Gold, M. H., V. L. Bradshaw, M. M. Boring, T. M. Bridges and J. A. Biron (2006) Split-face comparison of photodynamic therapy with 5 -aminolevulinic acid and intense pulsed light versus intense pulsed light alone for photodamage. Dermatol. Surg. 32, 795-801; discussion 801-3.

39. Sanclemente, G., L. Medina, J. F. Villa, L. M. Barrera and H. I. Garcia (2011) A prospective split-face double-blind randomized placebo-controlled trial to assess the efficacy of methyl aminolevulinate + red-light in patients with facial photodamage. J. Eur. Acad. Dermatol. Venereol. 25, 49-58.

40. Li, W., I. Yamada, K. Masumoto, Y. Ueda and K. Hashimoto (2010) Photodynamic therapy with intradermal administration of 5aminolevulinic acid for port-wine stains. J. Dermatolog. Treat. 21, 232-239.

41. Eiselein, L., D. W. Wilson, M. W. Lame and J. C. Rutledge (2007) Lipolysis products from triglyceride-rich lipoproteins increase endothelial permeability, perturb zonula occludens-1 and F-actin, and induce apoptosis. Am. J. Physiol. Heart Circ. Physiol. 292, H2745H2753.

42. Kidoya, H., H. Naito and N. Takakura (2010) Apelin induces enlarged and nonleaky blood vessels for functional recovery from ischemia. Blood 115, 3166-3174.

43. Ota, H., M. Matsumura, N. Miki and H. Minamitami (2009) Photochemically induced increase in endothelial permeability regulated by RhoA activation. Photochem. Photobiol. Sci. 8, 1401-1407.

44. Endlicher, E., P. Rummele, F. Hausmann, R. Krieg, R. Knuchel, H. C. Rath, J. Scholmerich and H. Messmann (2001) Protoporphyrin IX distribution following local application of 5-aminolevulinic acid and its esterified derivatives in the tissue layers of the normal rat colon. Br. J. Cancer 85, 1572-1576.

45. Juzeniene, A., P. Juzenas, L. W. Ma, V. Iani and J. Moan (2006) Topical application of 5-aminolaevulinic acid, methyl 5-aminolaevulinate and hexyl 5-aminolaevulinate on normal human skin. Br. J. Dermatol. 155, 791-799.

46. Marti, A., P. Jichlinski, N. Lange, J. P. Ballini, L. Guillou, H. J. Leisinger and P. Kucera (2003) Comparison of aminolevulinic acid and hexylester aminolevulinate induced protoporphyrin IX distribution in human bladder cancer. J. Urol. 170, 428-432.

47. Casas, A., H. Fukuda, G. Di Venosa and A. M. Batlle (2000) The influence of the vehicle on the synthesis of porphyrins after topical application of 5-aminolaevulinic acid. Implications in cutaneous photodynamic sensitization. Br. J. Dermatol. 143, 564-572.

48. Casas, A., C. Perotti, H. Fukuda, L. Rogers, A. R. Butler and A. Batlle (2001) ALA and ALA hexyl ester-induced porphyrin synthesis in chemically induced skin tumours: the role of different vehicles on improving photosensitization. Br. J. Cancer 85, 1794-1800. 